Check for updates

Cite this: Mater. Horiz., 2020, 7, 2479

Received 3rd July 2020

Accepted 21st August 2020

DOI: $10.1039 / \mathrm{d} 0 \mathrm{mh} 01085 \mathrm{e}$

rsc.li/materials-horizons

\section{A structural chemistry look at composites recycling}

\author{
Carlos A. Navarro, (D) a Cassondra R. Giffin, (D) a Boyang Zhang, (D) ${ }^{a}$ Zehan Yu, (D) b \\ Steven R. Nutt (iD ${ }^{b}$ and Travis J. Williams (iD *a
}

\begin{abstract}
Composite materials, especially carbon fiber-reinforced polymers (CFRPs), are high-performance class of structural materials now commonly used in aircraft, marine, and other applications, with emerging large-scale use in the automotive and civil engineering applications. The difficulty of recycling these materials is a key obstacle preventing their further application in larger markets. For decades, the engineering community has pursued physical methods to recover value from end-of-life composite waste. This work has generated scalable methods to recover modest value from CFRP waste, but because of their low value recovery, these are applied to a small fraction of CFRP waste. By contrast, relatively few methods to recycle CFRPs have been based on strategic approaches systematically to deconstruct the thermoset polymers that hold them together. In this Focus Article, we will show the emergence of these structure-focused approaches to CFRP recycling and illustrate the path of this research toward the ultimate realization of methods to recover both the reinforcing fibers and the thermoset materials that comprise modern CFRPs.
\end{abstract}

\section{Introduction}

Fiber-reinforced polymer (FRP) composites are structural materials that offer superior specific properties (strength and modulus), longer life, and increased efficiency compared to conventional structural metals. ${ }^{1}$ FRPs are now commonly used in aerospace, wind turbine, marine, and sporting goods applications, with emerging large-scale use in the automotive industry and some civil engineering applications. Most of the polymer matrices used in these materials are thermosets, frequently epoxies, and undergo polymerization in the manufacturing process to cure the resin from a viscous liquid into a stiff, glassy solid. The irreversibility of this process makes recycling FRPs challenging. The absence of a sustainable recycling pathway is an increasingly urgent problem impeding wider adoption of these materials.

FRP composites have become a primary structural material in the latest generation of commercial aircraft because they are lighter, more resistant to fatigue and corrosion, and reduce fuel consumption and maintenance when compared to structural metals. Uses include fuselage sections, wings, and control surfaces in aircraft such as the Boeing 787 Dreamliner and Airbus A350 XWB, which consist of more than $50 \%$ composite

\footnotetext{
${ }^{a}$ University of Southern California Loker Hydrocarbon Research Institute, 837 Bloom Walk, Los Angeles, CA, 90089, USA. E-mail: travisw@usc.edu

${ }^{b}$ University of Southern California M.C. Gill Composites Center,

3650 McClintock Ave, Los Angeles, CA, 90089, USA
}

parts by weight. FRPs are also important in the wind energy industry: glass fiber-reinforced polymers are currently the primary structural material in wind turbine blades. The wind industry is motivated to transition to carbon fiber for manufacturing larger turbines. With blade lengths now exceeding $100 \mathrm{~m}$, carbon fiber is used selectively in spar caps to provide the stiffness required to prevent column collisions under gust loads. Composites are also widely seen in high performance sporting goods, ranging from marine vessels to racing bicycles, golf shafts, skis, and hockey sticks. This range of applications is poised to expand. FRPs have also emerged as potential replacements for structural metals in high-volume automotive applications. Particularly, the BMW i3 and 7 series BEVs (battery electric vehicles) use FRPs to reduce weight and extend range, which help meet stringent standards for greenhouse gas emissions. In addition, FRPs are used for structural retrofitting, power transmission lines, and modular housing. While expanding use of FRPs reduces adverse environmental impact, particularly arising from fuel savings, these benefits are mitigated by the problem of FRP waste disposal.

There are currently no approaches for recycling end-of-life FRP composite products that can keep up with the volume of waste. Existing methods focus on recovering fibers by either (1) shredding the FRP and downcycling it to be an additive in materials like reinforced concrete or (2) pyrolyzing or dissolving the polymer matrix. These processes (Fig. 1, top) destroy the matrix and can damage the fibres, thus reducing their length, strength, and stiffness. This converts aligned fiber beds into lower-value random short-fiber mats. 


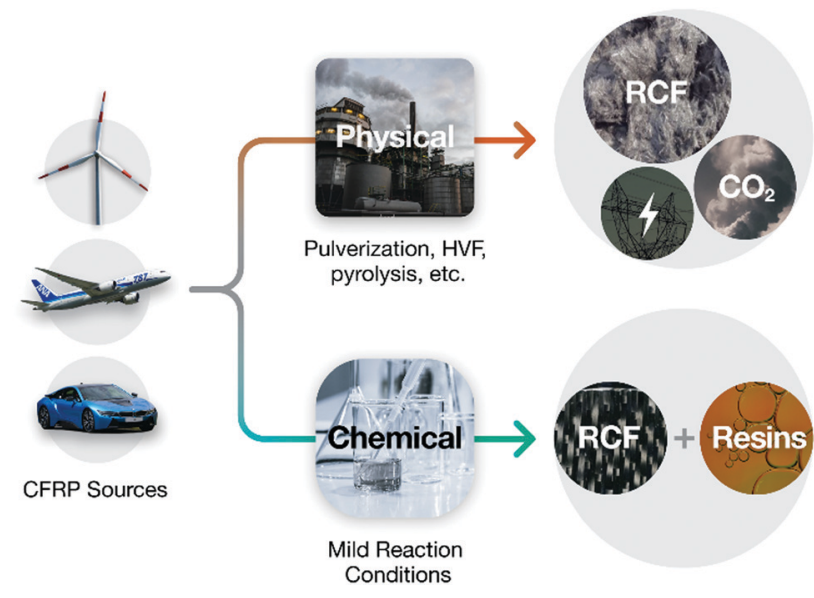

Fig. 1 Waste CFRP recycled using physical processes usually recover milled fibers by crushing or combusting the matrix, recovering its energy as heat or electricity, while mild chemical methods can recover woven fiber weaves and resin components.

Three decades of work in FRP composite recycling have not resulted in a tenable solution to the deconstruction and recycling of these materials, but methods are now beginning to appear that specifically target the chemical vulnerabilities of certain composite matrices (Fig. 1, bottom). Chemical recycling methods are diverse, ranging from using the unique properties of supercritical solvents to separate polymer matrices from their reinforcing fibres, to chemical reactions tailored for selective bond cleavage. Through these advancements, new products such as near-virgin quality fibers and useful organic small molecule and polymeric recyclates are being recovered for the first time.

Another emerging strategy for managing CFRP lifecycles is to build the deconstruction plan into the matrix at the point of its original manufacture. Popular strategies, such as vitrimers or covalent adaptable networks (CAN), function based on reversible, dynamic covalent bond exchange processes available with reactions like transesterification, Diels-Alder cycloadditions, reversible radical processes, and olefin metathesis. ${ }^{2}$ These materials can act as conventional thermosets under normal operating conditions, but when the bond exchange dynamics are fast relative to the duration of an external stimulus, such as heat, the polymer acts like a thermoplastic with high malleability and reprocessability. ${ }^{3,4}$ While such materials are not well-suited to the manufacture of components for heat-sensitive applications, this strategy can simplify matrix recycling and undoubtedly will be useful in some select applications. Since it's hard to imagine that we would fly in an airplane that is designed to deconstruct when it is heated, expanded efforts to recycle matrices found in current composites are clearly necessary.

Fiber-reinforced polymer composites are "an important cross-cutting technology" for U.S. manufacturing, ${ }^{5}$ with applications in transportation (aerospace, automotive), power generation, and infrastructure. The global composites industry is experiencing $c a$. $8 \%$ annual growth, leading up to a total volume of $\$ 131 \mathrm{~B}$ in 2024. Composites are emerging in automotive manufacturing; 45 billion pounds of composite materials were sold into the industry in $2019 .{ }^{6}$ Aerospace growth is predicted to continue across several application areas, and wind energy (blades) is slated for similar growth. ${ }^{7}$

By contrast to the industry's rapid growth, the present state of composites recycling is woeful: a mere $1 \%$ of CF (estimated) is recovered and reused as of 2020. This stems from key gaps in recycling technology: current recycling approaches cannot recover the resin or retain the fiber architecture/alignment/ continuity, relegating the reclaimed fiber to downcycling pathways. Leaders in the aerospace and automotive industries tell us that the absence of a reasonable recycling pathway for these materials at the end of their lives is a key factor impeding their more broad use. ${ }^{8,9}$ This creates strong push to develop new technology, and we see few firms in this space.

This Focus Article provides an overview of methods that are currently in use in FRP recycling, then show how the design of methods to target specific polymerization linkages in thermoset matrices can enable new progress in this area, ultimately to enable new chemical methods with which to approach the FRP recycling problem.

\section{Physical recycling methods}

Physical methods for CFRP recycling rely on size-reduction strategies like pulverization, where composite waste is mechanically shredded into pellets and added into new composites or cement as structural filler. ${ }^{10}$ There is merit in this approach: composite additives in construction materials like concrete are advantageous because the CFRP epoxy matrix improves bonding with the concrete, imparting improved ductility, load-carrying capacity, and fracture toughness. ${ }^{11}$ Although pulverization fully re-uses the waste composite, the value obtained by using CFRP material as an additive is minute compared to the initial financial and energetic costs used to manufacture the starting fibers. Carbon fibers are estimated to cost up to $\$ 30 \mathrm{USD} \mathrm{lb}^{-1}$ and require up to $75 \mathrm{~kW} \mathrm{~h} \mathrm{lb}{ }^{-1}$ to produce, while reinforced concrete costs about \$0.17 USD $\mathrm{lb}^{-1} \cdot \cdot^{12,13}$ While this approach preserves some value, much value remains to be recovered if the carbon fiber weaves could be preserved for high-quality reuse.

There's environmental merit in this technology because composites that are shredded and used as building materials do not go immediately to landfill. Globally, $<1 \%$ of composite waste is recycled, and even if it is significantly downcycled into a single-use material like concrete, some of its in-service life can be preserved and landfill impact can be reduced. As more composite waste is recycled, presumably using increasingly value-preserving technologies, this downcycling method will retain value, because both the volume of unutilized waste is large, and downcycling by shredding can impart a second life to materials that will remain refractory to modern methods.

Alternative strategies to pulverization have been developed that can separate polymer matrix from carbon fiber. One example is high voltage fragmentation (HVF), a method in which the 


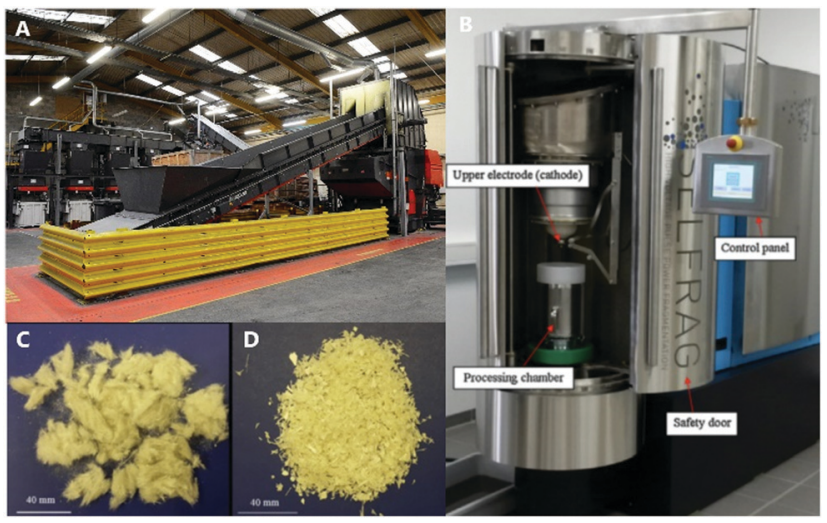

Fig. 2 (A) A commercial mechanical recycling instrument, (B) a high voltage fragmentation instrument, (C) glass fibers recovered using high voltage fragmentation, (D) glass fibers recovered from mechanical recycling. Images borrowed with permission. ${ }^{14}$

composite is immersed in water and repeatedly pulsed with electrical discharge (Fig. 2). This creates extreme temperatures and pressures at the composite surface that disintegrate the matrix. ${ }^{14}$ However, the treatment time necessary to recover fibers without residue is too long to be practical and reduces the average fiber length. ${ }^{14}$ In an alternative to pyrolysis, fluidised bed processes (FBPs) were developed where hot air passes through a silica bed containing shredded composites to remove the matrix from the fiber. ${ }^{1}$ The matrix particles are carried away by the air stream to a separate chamber for destruction at $1000{ }^{\circ} \mathrm{C}^{1}{ }^{1} \mathrm{FBPs}$ have the same limitations as HVF in that the matrix is destroyed, and the fibers are disordered, which highlights two key opportunities for improving composite recycling.

Generally, these methods are analogous to pyrolysis in that the polymer matrix is discarded and the fibers are downgraded, sacrificing fiber continuity and fiber architecture. Depending on the approach, however, much higher quality fibers can be retained and re-manufactured into higher-value products, such as moulding compounds, than are possible with pyrolysis recyclates or reinforced concrete.

\section{High-pressure decomposition}

High-pressure methods for recycling composites typically rely on a solvent system, sometimes with an acid or base reagent, that is heated and pressurized to become supercritical. Supercritical fluids have unusual properties, including low viscosity, high diffusivity, and increased solvation strength, and these qualities are appealing for composite recycling because they better permeate the material and accelerate matrix dissolution. ${ }^{15}$ Supercritical solvents can facilitate bond cleavage within polyester and amine-cured composites that are inert under other conditions. Thus, high-pressure recycling has become an important area of investigation, allowing recovery of carbon fibers with excellent physical properties using inexpensive, non-toxic, and recyclable reagents. ${ }^{16}$ Because of the diversity of reagents and conditions used in this general strategy, the quality of the fibers varies widely. More chemically mild approaches enable recovery of relatively undamaged fibers, as is the case in hydrolysis of polyester-based matrices. Other conditions that require strongly oxidative or corrosive conditions can damage or cleave fibers and downgrade them to applications such as bulk moulding compounds. This highlights the general conundrum of aerospace-grade resins: the conditions required to disassemble more robust thermoset polymers used in more demanding applications also tend to degrade their imbedded fibers.

Common solvents used in supercritical CFRP recycling include water, short chain alcohols and ketones, or a mixture of these. Mixed solvent systems have the benefit of reducing the supercritical temperature and pressure thresholds and increasing the matrix by-product solubility. ${ }^{9}$ Supercritical solutions of water have successfully removed $>95 \%$ of amine-cured epoxy resins from fibers in as little as 15 minutes, yielding derivatized monomers like methylenedianilines and biphenyldiamines. ${ }^{17}$ Based on by-product analysis, these conditions appear to target crosslinked $\mathrm{C}-\mathrm{N}$ bonds and secondary alcohols, and the observation of diaminobenzophenone species implies there is an oxidant, likely oxygen gas, performing $\mathrm{C}-\mathrm{H}$ and oxidations (Scheme 1). ${ }^{17,18}$ Other solutions like $80 \%$ acetone in water, $20 \%$ butanone in water, or neat propylene glycol have also been effectively used for dissolving amine- and anhydride-cured epoxy matrices. ${ }^{19-21}$

A variety of chemical additives have been studied to accelerate resin dissolution, frequently by changing the dissolution mechanism. Hydroxide salts added to supercritical alcohol solutions remove $70 \%$ more matrix from amine-cured composites than control experiments. ${ }^{22}$ The molecular mechanism for this is not obvious. The same process has been adapted from a batch reaction to a semi-continuous process that enables recovery of resin-free fibers at conditions $100{ }^{\circ} \mathrm{C}$ less forcing and $10 \mathrm{MPa}$ less forcing than those of the batch process. This is realized by reducing mass transfer limitations, showcasing the

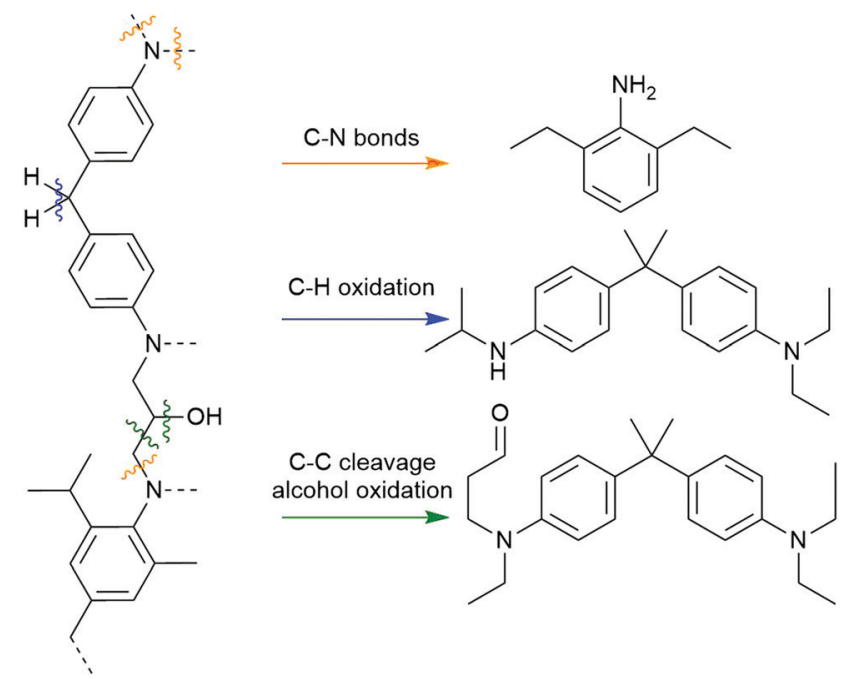

Scheme 1 Bonds within amine-cured epoxies which are experimentally found to break when exposed to supercritical water and alcohols. ${ }^{8}$ 
impact of clever reactor design on CFRP recycling. ${ }^{22}$ Liu et al. added phenol and potassium hydroxide to supercritical water, and observed improvement of more than $80 \mathrm{wt} \%$ in resin removal. They attributed this success to phenxoyl-based freeradical reactions. ${ }^{23}$ Adding ionic liquids to ethylene glycol shifts the product distribution away from long-chain oligomers to monomers in polyester matrix transesterification processes. ${ }^{24}$ Despite the benefits of chemical additives, scaling up high-pressure recycling is inherently difficult due to the high energy demand, safety considerations, and need for specialized reactors. ${ }^{25}$

\section{Atmospheric pressure decomposition}

The introduction of mild conditions to selectively depolymerize FRP matrices opens new possibilities in FRP recycling, because such conditions enable fiber recovery with less damage, sometimes even retaining their original weave, and fine chemicals that form from an orderly deconstruction of the composite matrix. Chemical recycling methods that have been developed near ambient pressure also tend to be safer and easier to implement at an industrial scale than high-pressure methods, increasing their likelihood of adoption. ${ }^{26}$ However, without the brute force provided by supercritical temperatures and pressures, these reactions must rely on grace and design to select chemical reagents to cleave crosslinking bonds in the matrix: this fundamentally changes FRP recycling from an engineering problem to a chemistry problem.

Designing systems for CFRP recycling that target specific features of the network of their thermoset polymers at mild conditions requires expertise in basic reaction chemistry, which has not previously been a focus of the composites recycling community. As new chemistries are introduced, however, milder and more delicate conditions are emerging. As a result, we are seeing cases now where polymers and fine chemicals can be collected while simultaneously recovering fibers. Unfortunately, any recycling approach based on a specific polymer structure or formulation will probably be limited in use to CFRPs containing that same (or analogous) linking chemistry. Thus, the problem becomes complex, and the continued relevance for bulk physical methods is highlighted as one considers the problem of unknown or mixed composite waste streams.

When engineering these new chemical processes, conditions used should be inexpensive, selective, safe to handle on large scale, and yield only benign by-products. It is likely that a reason that the community has seen so few is that processminded thinkers have set aside possible but impractical methods that could homogenize thermoset matrices, but are irrelevant because of reagent cost, safety, or other practical concerns. Some emerging examples of successful chemical methods follow.

The crosslinking polyester bonds in anhydride-cured epoxy composites are susceptible to acid- and base-catalyzed transesterifications, which has been exploited to great success. Strong acids like $p$-toluenesulfonic acid in acetic anhydride solution can homogenize polyester resin blocks at temperatures as low as $80{ }^{\circ} \mathrm{C}$, though other bonds, such as the quaternary carbon in

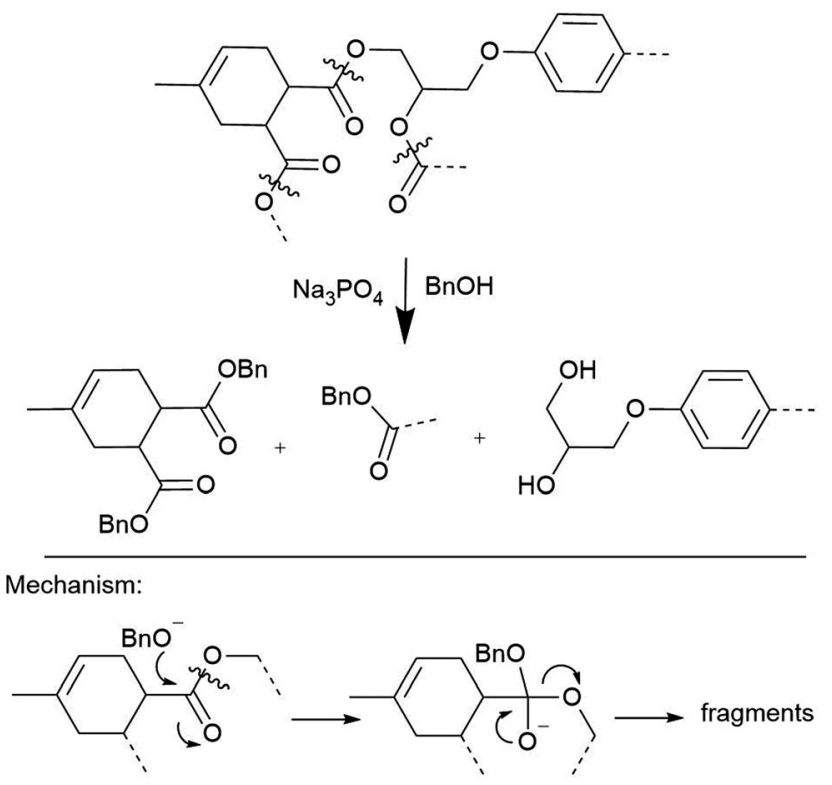

Scheme 2 Hitachi's transesterification conditions for depolymerization of acid-anhydride linked FRP resins. ${ }^{31}$

bisphenol A moieties, are cleaved as well. ${ }^{27}$ Short-chain alcohol solutions containing Lewis bases like hydroxides or amines degrade these matrices in as little as 90 min. ${ }^{28-30}$ Derivatized monomers and high-quality recycled carbon fibers are usually recovered for re-use from these reactions, as Hitachi Chemical demonstrated. ${ }^{31,32}$ The inherent lability of the ester, the linker formed upon curing anhydride-cured epoxy resin system, is a feature of these methods that contributes to their success: the lability of the ester serves almost like an engineered strategy for polymer disassembly (Scheme 2). Unfortunately, programming this or any other designed vulnerability into the CFRP matrix will limit the material's use cases, because it will have a known vulnerability. There are, however, exceptions, such as efforts to incorporate cleavable amines into epoxies that facilitate deconstruction. $^{33}$

Unlike esters, or even amides, that are amenable to acidic or alkaline solvolysis, epoxy thermosets do not contain labile bonds that enable facile disassembly. The carbon-nitrogen bonds that impart stability and rigidity to amine-cured epoxies are comparatively stable to acids and bases, so researchers have used more forcing conditions to realize less complete dissolution than is realized with anhydride-cured matrices. For example, an amine-cured resin block must be soaked in nitric acid for 21 days for degradation to occur. ${ }^{34}$ Solutions containing excess Lewis acids, like zinc or aluminum chlorides, have been used successfully to digest composites, however, the molecular mechanism of these degradation reactions remains unclear. ${ }^{35,36}$ One group suggests that aluminum coordination to Lewis basic sites in the CFRP matrix creates a leaving group, but this seems uncertain, as aluminum salts other than the chloride fail to catalyze the reaction. ${ }^{36}$ Basic conditions are similarly forceful and unsuccessful: imidazole-cured epoxy Novolac matrices require 2 hours in molten potassium hydroxide to homogenize. ${ }^{37}$ If the composite 

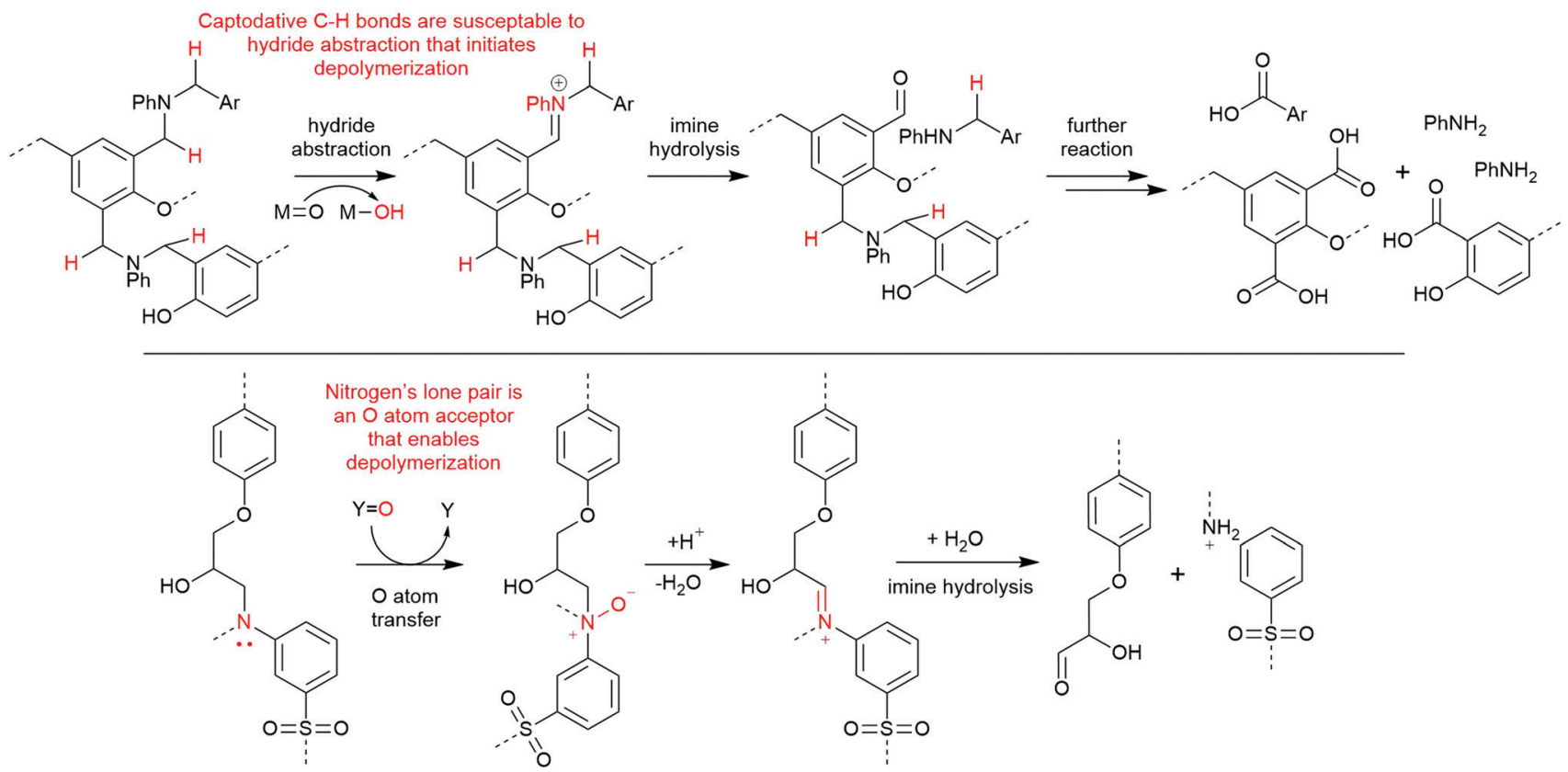

Scheme 3 Matrices cross-linked at nitrogen can be cleaved by oxidizing it to an hydrolyzable imine though (top) hydride abstraction in benzoxazine using high valent metal-oxo species, or (bottom) oxygen atom transfer under acidic conditions using hydrogen peroxide to form $\mathrm{N}$-oxides.

is swelled in nitric acid first, solutions of potassium hydroxide in poly(ethylene glycol) can eventually fully degrade the resin. ${ }^{38}$

Alternative chemical strategies are needed to recycle aminecured epoxy composites mildly and selectively. Their carbonnitrogen bond presents a potential target for selective cleavage, possibly by conversion to an $\mathrm{N}$-oxide or iminium cation, as the latter is readily cleaved by water. ${ }^{39}$ An analogous strategy was successfully utilized in depolymerizing benzoxazine-epoxy composites by abstracting a hydride atom alpha to an aniline nitrogen center using a high valent ruthenium species (Scheme 3, top). ${ }^{40}$ Another method is to use an inexpensive peroxide, like hydrogen peroxide, as an oxygen atom transfer agent to form an amine oxide, or $N$-oxide (Scheme 3, bottom). ${ }^{41,42}$ We have previously reported the formation of imines from $\mathrm{N}$-oxides via NMR spectroscopy in our degradation studies of small molecule analogues of amine-cured epoxies. ${ }^{43}$ Through this molecular study, we argue that other successful reports of amine-cured epoxy resin degradation involving oxidants like hydrogen peroxide misattribute the mechanism to a series of hydroxyl radical-based processes. ${ }^{44-46}$ Identifying the most-likely mechanism occurring during these degradation reactions is key to build on this knowledge as the community develops an optimal process for depolymerizing amine-cured epoxies.

There seems to be great promise in this general strategy of designing processes for selective depolymerization of CFRP thermoset matrices, but there are intrinsic limits to the approach. Structure-dependent recycling strategies will almost certainly be ineffective on polymers for which they were not designed. Using such a method would require the waste processor to know the basic composition of the waste stream. Further, the practical limitations of safety and cost must be superimposed on any of these methods before they can be scaled. Reagents in this literature such as nitric acid, hydrogen peroxide, certain expensive solvents, and even $\mathrm{O}_{2}$ itself impose cost and safety limits on the deployment of such processes. Much chemistry and engineering remains to be done to overcome these hurdles.

\section{Thermoset matrices with inherent recyclability}

Developing thermoset matrices with inherent recyclability, which allows them to be easily removed or recycled, is another attractive approach to composite recycling. Introducing labile chemical bonds and CANs (covalent adaptable networks) are two popular strategies to achieve this. By introducing degradable chemical bonds (such as the ester links), thermoset matrices can become recyclable when exposed to external stimuli, such as temperature, chemicals, or photolysis. ${ }^{47} \mathrm{~A}$ review of potential recyclable thermosets for structural applications and limitations thereof has appeared recently. ${ }^{47}$ A key problem of using degradable chemical bonds to modify thermoset matrices to increase recyclability is that the matrix structure is destroyed after degradation, which eliminates the value of the recyclates. CANs overcome this problem, as they retain the overall structure of the matrix after recycling. CANs can be categorized into two subgroups - dissociative and associative - based on the chemical mechanisms involved: original chemical bonds are broken either before or after the formation of new chemical bonds (Fig. 3).

Introducing thermally triggered degradable chemical bonds can reduce the degradation temperature. For example, by adding thermally cleavable carbamate bonds, redesigned cycloaliphatic 


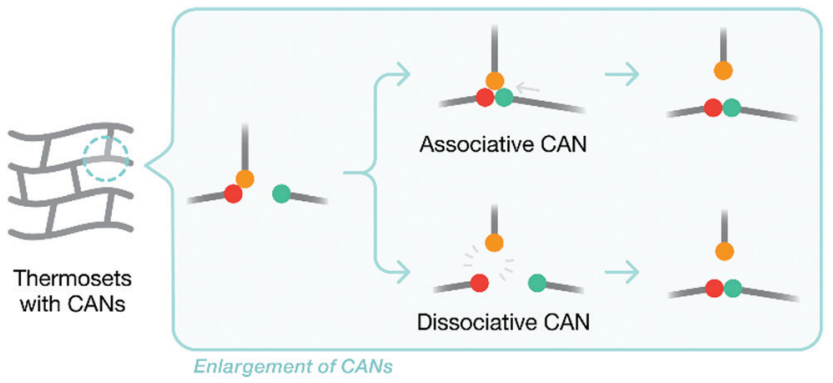

Fig. 3 A generalized diagram demonstrating the two pathways by which new bonds are formed between different functional groups in a CAN.

diepoxides were decomposed between 200-300 ${ }^{\circ} \mathrm{C}$, whereas commercial cycloaliphatic diepoxides are normally stable up to $350{ }^{\circ} \mathrm{C} .{ }^{48}$ Ester bonds are also frequently used to increase thermal reworkability. One example features the use of hyperbranched polyaminoester (PAE) with secondary alkyl esters as an additive to modify a conventional epoxy resin (DGEBA). The additive reduced the decomposition temperature up to $100{ }^{\circ} \mathrm{C}$. ${ }^{49}$

Some recyclable thermoset CANs can be triggered by specific chemical reagents. Early work on associative CANs utilized photoinitiated radical chain transfer reactions. ${ }^{50}$ More recent examples include a proprietary epoxy resin system called "Cleavamine," which contains acid-labile formyl and acetal groups (Fig. 4). ${ }^{51}$ This recyclable resin exhibits thermal and mechanical properties similar to non-recyclable resins, and this resin is easily degraded in an acidic environment. ${ }^{33}$ In this mechanism an ether group is protonated, leaves as an alcohol, and is replaced by water to form a hemiacetal. The second ether group is similarly protonated and leaves, yielding a ketone.

A second example is based on the Diels-Alder reaction, combining multi-furan and multi-maleimide polymers to form a novel, dynamic material (Fig. 5). ${ }^{52}$ The polymer displayed mechanical properties similar to commercial epoxy resins with the added advantage of self-healing. Structural failure was remediable after thermal treatment at $120-150{ }^{\circ} \mathrm{C}$ for about two hours.

Leibler and co-workers undertook pioneering work based on associative CANs, which involved a new group of material called vitrimers. ${ }^{53}$ Via transesterification reactions with proper catalysts, the viscosity of these vitrimers decreased only slightly with increasing temperature, whereas viscosity of conventional polymers changes rapidly near the glass transition temperature.

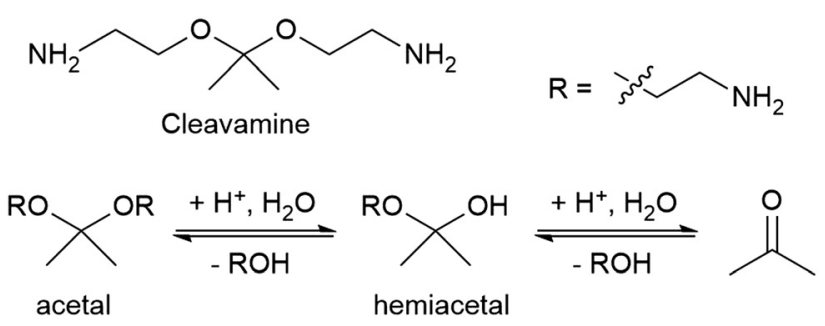

Fig. 4 Structure of a cleavamine curing agent containing a central acetal group and the reaction of its acid hydrolysis into a ketone and two amino alcohols. ${ }^{33}$

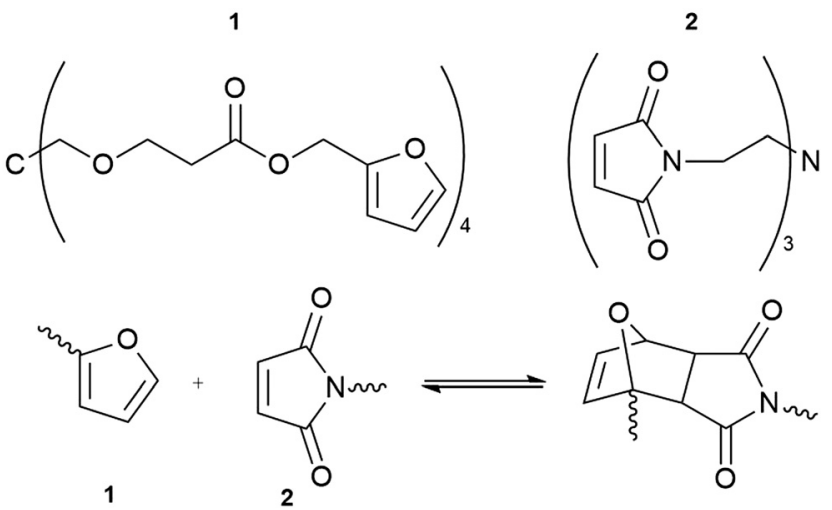

Fig. 5 The reversibility of the Diels-Alder cycloaddition reaction between multi-diene 1 and multi-dienophile 2 allows the resulting polymer to repair cracks without requiring additional reagents to catalysts. ${ }^{52}$

\section{Overview}

Physical methods to reclaim value from composite materials have created an avenue to deflect composite waste from landfill and re-insert waste materials into consumer products. However, the downcycling of value in such processes has limited their commercialization such that these methods reclaim only a small portion of composite waste. A second generation of approaches is now emerging in which chemical strategies are being designed selectively to deconstruct the bonds that hold together composite thermoset matrices. Such strategies, along with new composite resin technology in which cleavable bonds are built into the polymer when it is first produced, both move the composite materials industry toward sustainability and highlight the vital role that synthetic chemistry must play in enabling fundamental advancement in composites engineering.

The present landscape for composite recycling includes multiple approaches, each with intrinsic drawbacks and advantages, providing fertile ground for opinion and speculation about a path forward. A practical criterion for comparing recycling approaches should weigh both sustainability and economic factors. Clearly, any approach that destroys the fiber architecture and continuity must either (a) include realignment of fibers and the attendant cost, or (b) be considered a downcycling approach. Downcycling to lower-performance products reclaims fibers for insertion into a second application, but postpones recycling to the end of the second service life. Such approaches also sacrifice the matrix, a highly engineered material of value not unlike that of the fibers. While such approaches do not require new technology and may thus be economically viable, they are not preferred as a long-term solution. Chemical approaches that can reclaim both fibers and matrix components offer a superior solution, provided they operate at acceptable rates and costs, do not create additional recycling problems, and selectively cleave the matrix polymers such that high-value molecular components are retained. Chemical solutions avoid mechanical shredding and retain fiber architecture, avoiding major downstream costs. However, the challenge with chemical processes lies in finding a scalable solution that requires only relatively mild conditions. 
Finally, all approaches face a major issue - lack of market pull and until this issue is addressed, progress is likely to be modest in the foreseeable future. Eventually, the waste disposal problem will become so severe that legislation will be introduced to pressure consumers and producers to adopt more sustainable practices.

\section{Conflicts of interest}

Navarro and Williams are part of a startup company, Closed Composites LLC, that is working to commercialize catalytic methods to recycle CFRPs, including some described here.

\section{Acknowledgements}

This work is sponsored by the US Department of Defense (SERDP WP20-1491) and USC through the Stevens Center for Innovation, MC Gill Composites Center, and Loker Hydrocarbon Research Institute. Fellowship assistance from USC Dornsife College (C. R. G.) and Provost (B. Z.) is gratefully acknowledged.

\section{Notes and references}

1 S. Karuppannan Gopalraj and T. Kärki, SN Appl. Sci., 2020, 2, 433.

2 C. J. Kloxin and C. N. Bowman, Chem. Soc. Rev., 2013, 42, 7161-7173.

3 K. Yu, Q. Shi, M. L. Dunn, T. Wang and H. J. Qi, Adv. Funct. Mater., 2016, 26, 6098-6106.

4 P. Taynton, H. Ni, C. Zhu, K. Yu, S. Loob, Y. Jin, H. J. Qi and W. Zhang, Adv. Mater., 2016, 28, 2904-2909.

5 United States Department of Energy, An Assessment of Energy Technologies and Research opportunities, 2015.

6 S. Mazumdar, D. Pichler, H. GangaRao, M. Benevento, R. Liang and E. Witten, Compos. Manuf., 2019, http://compo sitesmanufacturingmagazine.com/2019/01/2019-state-of-theindustry-report/10/.

7 United States Department of Energy, Bandwidth Study on Energy Use and Potential Energy Saving Opportunities in U.S. Carbon Fiber Reinforced Polymer Manufacturing, 2017.

8 D. Brosius, Composites World, 2019.

9 G. Oliveux, L. O. Dandy and G. A. Leeke, Prog. Mater. Sci., 2015, 72, 61-99.

10 G. Giulvezan and B. Carberry, Boeing Environmental Technotes, 2003, 8, 1-4.

11 L. W. Zhang, A. O. Sojobi and K. M. Liew, J. Cleaner Prod., 2019, 233, 56-75.

12 E. Asmatulu, M. Overcash and J. Twomey, J. Ind. Eng., 2013, 2013, 1-8.

13 HomeGuide, Concrete Prices, https:/homeguide.com/costs/ concrete-prices\#reinforcement, accessed 06/26/20, 2020.

14 P. T. Mativenga, N. A. Shuaib, J. Howarth, F. Pestalozzi and J. Woidasky, CIRP Ann., 2016, 65, 45-48.

15 M. Goto, J. Supercrit. Fluids, 2009, 47, 500-507.

16 C. Morin, A. Loppinet-Serani, F. Cansell and C. Aymonier, J. Supercrit. Fluids, 2012, 66, 232-240.
17 L. Henry, A. Schneller, J. Doerfler, W. M. Mueller, C. Aymonier and S. Horn, Polym. Degrad. Stab., 2016, 133, 264-274.

18 Y. Li and S. Wang, in Advanced Supercritical Fluids Technologies, ed. I. Pioro, IntechOpen, 2019, ch. 6, , DOI: 10.5772/ intechopen.89591.

19 G. Oliveux, J.-L. Bailleul, A. Gillet, O. Mantaux and G. A. Leeke, Compos. Sci. Technol., 2017, 139, 99-108.

20 G. Tesoro and Y. Wu, Adv. Polym. Technol., 1993, 12, 185-196.

21 K. H. Yoon, A. T. DiBenedetto and S. J. Huang, Polymer, 1997, 38, 2281-2285.

22 R. Piñero-Hernanz, J. García-Serna, C. Dodds, J. Hyde, M. Poliakoff, M. J. Cocero, S. Kingman, S. Pickering and E. Lester, J. Supercrit. Fluids, 2008, 46, 83-92.

23 Y. Liu, J. Liu, Z. Jiang and T. Tang, Polym. Degrad. Stab., 2012, 97, 214-220.

24 H. Wang, Y. Liu, Z. Li, X. Zhang, S. Zhang and Y. Zhang, Eur. Polym. J., 2009, 45, 1535-1544.

25 S. Pimenta and S. T. Pinho, Waste Manag, 2011, 31, 378-392. 26 J. A. Romagnoli, Introduction to Process Control, 2005.

27 Y.-Q. Lei, Z.-X. He, Y. Luo, S.-N. Lu and C.-J. Li, Polym. Degrad. Stab., 2016, 123, 115-120.

28 P. Yang, Q. Zhou, X.-X. Yuan, J. M. N. van Kasteren and Y.-Z. Wang, Polym. Degrad. Stab., 2012, 97, 1101-1106.

29 X. Kuang, Y. Zhou, Q. Shi, T. Wang and H. J. Qi, ACS Sustainable Chem. Eng., 2018, 6, 9189-9197.

30 K. El Gersifi, G. Durand and G. Tersac, Polym. Degrad. Stab., 2006, 91, 690-702.

31 M. Nakagawa, K. Kasuga, K. Aoyagi, K. Ishihara, Y. Ikeda and K. Shibata, in Proceedings of the American Society for Composites 2014-Twenty-ninth Technical Conference on Composite Materials, DEStech Publications, 2014, ch. Session IX: Sustainability/Efficiency in Composite Processing, pp. 34-52.

32 M. Nakagawa, H. Kuriya and K. Shibata, presented in part at the 5th International Symposium on Feedstock Recycling of Polymeric Materials, Chengdu, China, 2009.

33 M.-S. Wu, C. B. Jin, X. Li and S. Nutt, Adv. Manuf.: Polym. Compos. Sci., 2019, 5, 114-127.

34 W. Dang, M. Kubouchi, S. Yamamoto, H. Sembokuya and K. Tsuda, Polymer, 2002, 43, 2953-2958.

35 T. Liu, M. Zhang, X. Guo, C. Liu, T. Liu, J. Xin and J. Zhang, Polym. Degrad. Stab., 2017, 139, 20-27.

36 Y. Wang, X. Cui, H. Ge, Y. Yang, Y. Wang, C. Zhang, J. Li, T. Deng, Z. Qin and X. Hou, ACS Sustainable Chem. Eng., 2015, 3, 3332-3337.

37 W. Nie, J. Liu, W. Liu, J. Wang and T. Tang, Polym. Degrad. Stab., 2015, 111, 247-256.

38 J. Jiang, G. Deng, X. Chen, X. Gao, Q. Guo, C. Xu and L. Zhou, Compos. Sci. Technol., 2017, 151, 243-251.

39 E. H. Cordes and W. P. Jencks, J. Am. Chem. Soc., 1963, 85, 2843-2848.

40 J. N. Lo, S. R. Nutt and T. J. Williams, ACS Sustainable Chem. Eng., 2018, 6, 7227-7231.

41 R. D. Bach, M. Su and H. B. Schlegel, J. Am. Chem. Soc., 1994, 116, 5379-5391. 
42 Y. Ma and S. Nutt, Polym. Degrad. Stab., 2018, 153, 307-317.

43 C. A. Navarro, E. A. Kedzie, Y. Ma, K. H. Michael, S. R. Nutt and T. J. Williams, Top. Catal., 2018, 61, 704-709.

44 M. Das and S. Varughese, ACS Sustainable Chem. Eng., 2016, 4, 2080-2087.

45 J. Li, P.-L. Xu, Y.-K. Zhu, J.-P. Ding, L.-X. Xue and Y.-Z. Wang, Green Chem., 2012, 14, 3260-3263.

46 P. Xu, J. Li and J. Ding, Compos. Sci. Technol., 2013, 82, 54-59.

47 W. Post, A. Susa, R. Blaauw, K. Molenveld and R. J. I. Knoop, Polym. Rev., 2020, 60, 359-388.
48 M. Morell, M. Erber, X. Ramis, F. Ferrando, B. Voit and A. Serra, Eur. Polym. J., 2010, 46, 1498-1509.

49 M. Morell, X. Fernández-Francos, X. Ramis and A. Serra, Macromol. Chem. Phys., 2010, 211, 1879-1889.

50 D. Montarnal, M. Capelot, F. Tournilhac and L. Leibler, Science, 2011, 334, 965-968.

51 Adesso Advanced Materials, Recyclable curing agent, http:// www.adessomaterials.com/products.aspx? classid $=30$.

52 X. Chen, M. A. Dam, K. Ono, A. Mal, H. Shen, S. R. Nutt, K. Sheran and F. Wudl, Science, 2002, 295, 1698-1702.

53 T. F. Scott, A. D. Schneider, W. D. Cook and C. N. Bowman, Science, 2005, 308, 1615-1617. 